Journal of Engineering and Science Research 4 (1): 40-46, 2020

e-ISSN: 2289-7127

(C) RMP Publications, 2020

DOI: $10.26666 / \mathrm{rmp}$.jesr.2020.1.7

\title{
Short Term Effect of Virtual Reality on Tear Film Stability and Ocular Discomfort
}

\author{
Mohamad Syarifuddin Sidik Ahmad ${ }^{1}$, Mohd Zulfaezal Che Azemin ${ }^{1}$, Mohd Hafidz Ithnin ${ }^{1}$ and \\ Mohd Izzuddin Mohd Tamrin ${ }^{2}$ \\ ${ }^{1}$ Kulliyyah of Allied Health Sciences, International Islamic University Malaysia, \\ Bandar Indera Mahkota, 25200 Kuantan, Malaysia. \\ ${ }^{2}$ Kulliyyah of ICT, International Islamic University Malaysia, Gombak, 53100 Kuala Lumpur, \\ Malaysia.
}

\begin{abstract}
Virtual reality (VR) has been integrated and used with smartphones as one of the digital entertainments such as in gaming and movie streaming. With emergent of various VR brands in the market, it concerns the public on the possible side effects of VR on the ocular performance specifically on tear film stability and ocular discomfort. The purpose of this study was to compare the change on non-invasive keratograph tear break-up time (NIKBUT), tear meniscus height (TMH) before and after the use of VR for 30 minutes, and to measure the ocular discomfort after the use of VR quantitatively. Thirty-two subjects were recruited in this study and all the subjects were divided randomly into two groups; VR and laptop (used as a control) groups. Each subject needed to watch a movie for 30 minutes using the respective devices. The changes of tear film stability and ocular discomfort before and after the use of the devices were then compared and the results showed that there was no significant difference ( $p>0.05$ ) after 30 minutes of the use. Comparison of the ocular parameters between the devices on post-watching activities was also not significant ( $p>0.05$ ). Use of VR did not give any signs of dryness to the eyes and it can be used without any discomfort even after 30 minutes usage of the devices.
\end{abstract}

Key words: Virtual reality, Tear film stability, Tear break-up time, Tear meniscus height, Ocular discomfort

\section{INTRODUCTION}

Virtual reality (VR) is one of the latest technological achievements invented for the human eyes which give a different level of visual experience to the users. It allows human to interact with the computer-generated image which projects 3-dimensionals (3D) virtual environment. This paper [1] defined the VR as a "realtime interactive graphics with 3D models, combined with a display technology that gives the user the immersion in the model world and direct manipulation". With head-mounted VR devices (HMD) the generated dichoptic images will create a sense of presence that will interact with the user's head movements in the real world. Theoretically, the fixed images presented inside the HMD helmet, are very close in front of each eye which are viewed through powerful convex lenses, which will make the images appear to be located at a distance [2]. The imposition of relative lateral offset within the presented images creates image disparity on the retina which in the end produces 3D depth perception [2]. Currently, VR is developed in a variety and wider scope of applications such as in education, entertainment, medication, military, engineering and design industries [3]. It is also integrated specifically for the head mounted devices (HMD) and the smartphones available in the market, such as for 3D gaming and movie streaming.

VR popularity has increased significantly among smartphone users around the world and it is currently manufactured as consumer-grade HMD which is inexpensive and affordable. The users concerned with the effects of VR possibly to the eye performances and its comfort [4]. It has been claimed that it can cause similar symptoms to those reported with the use of visual display unit (VDU) which includes desktop, smartphones and other digital screen device [5], known

Corresponding Author: Mohd Zulfaezal Che Azemin, Kulliyyah of Allied Health Sciences, International Islamic University Malaysia, Bandar Indera Mahkota, 25200 Kuantan, Malaysia, email: zulfaezal@iium.edu.my. 
as Computer Vision Syndromes (CVS). It is used to describe the symptoms of eye and vision-related problems resulting from prolonged VDU exposure [6]. More than 80 percent of HMD users claimed to have simulator sickness symptoms including headaches, nausea, dizziness, and eye strain caused by HMD's usage [4]. The use of digital screen also directly had been related to dryness of the eye as it can affect blink patterns, ocular surface homeostasis and tear film function [7], which will contribute to a variety of other ocular discomfort symptoms.

Previously, there are not many studies conducted to explore the effects of 3D image visualization on tear stability and ocular discomfort as most of the studies limited their investigation to 2D. It is believed that blinking rate and near work position was one of the causes that lead to decrement of tear stability in 2D screen [8]. The same concept on its effects to the eye is applied to the head mounted VR, which is aggravated by closer viewing distance, and more wearing hours. The earliest studies of VR's side effect were from [9, $10,11]$. The data were insufficient to give any conclusion related with VR effect on the ocular performance. The current VR technology which incorporate $3 \mathrm{D}$ environment and improved display specifications might give different result to the tear film stability compared with the VR technology used in previous studies.

\section{METHODS AND MATERIALS}

The study has been approved by Kulliyyah Allied Health Sciences (KAHS) Ethics Committee (KAHS 71/18). Thirty-two participants aged 18-25 years old, were randomly recruited among the International Islamic University Malaysia population throughout the study period from September 2018 to March 2019.

Participants were provided with an informed consent forms containing information about the screening and the experiment, and about the possible occurrence of visual discomfort and visual fatigue. History taking and preliminary examination were also taken to meet the exclusion and inclusion criteria of the study. The selected subjects must be healthy with the best corrected vision (6/6). The participants also must not have dry eye (NIKBUT > 5 seconds), no corneal disease and abnormalities, such as keratoconus, no history of ocular refractive surgery and not wearing contact lens at least 24 hours before the procedures. The participants were selected based on the preliminary examination and clinical records. Prior the examination, 32 participants were randomly divided into two group: control and experimental groups. The control group used laptop, while experimental group used Samsung Gear VR 2017 headset. For each session, the NIKBUT before and after the usage of the device was measured respectively. Each group was asked to watch the same movie, titled 'How to Train Your Dragons 2' for 30 minutes but with different visual experience for each group, either 3D or 2D.

The ocular tear stability parameters involved in this study were non-invasive keratograph tear break up time (NIKBUT) and tear meniscus height (TMH) before and after the use of VR and laptop. The parameters were measured using Oculus Keratograph $5 \mathrm{M}(\mathrm{OK} 5 \mathrm{M})$. Normal NIKBUT value is $9.7 \pm 6.7$ seconds and $4.6 \pm 1.3$ seconds for dry eyes [12], while, the normal TMH is $0.20 \pm 0.05 \mathrm{~mm}$ and $0.14 \pm 0.03$ $\mathrm{mm}$ for the dry eye patient [13]. The measurement of NIKBUT and TMH were done on each eye individually for $3 \mathrm{D}$ and $2 \mathrm{D}$ visual experiences. Thus, for each visit, there would be two measurements of NIKBUT and TMH taken for each eye and the comparison of the mean of both parameters before and after the usage of devices were made specifically for each eye and each visual experience. Subjects also needed to fill the Simulator Sickness Questionnaire (SSQ) before and after the NIKBUT assessment for ocular discomfort measurement in both sessions.

SSQ is a widely used questionnaire for the measurement of psychological visual discomfort and user's perceived annoyance. Referring to [14], it has questions related with the discomfort symptoms and they are divided into 3 subcategories which are, nausea $(\mathrm{N})$, oculomotor $(\mathrm{O})$, and disorientation (D). The rating of comfort are none, slight, moderate and severe with the score of $0,1,2$, and 3 respectively. The sum of each subcategories, $\mathrm{N}, \mathrm{O}$ and $\mathrm{D}$ will be multiplied with their factor, which are $9.45,7.45$ and 13.36 respectively [14]. The sum of subcategories also will be multiplied with the factor of 3.46 to get finalized total score which is SSQ-Total [14]. The total SSQ will be used as a quantitative measurement to calculate the general 
visual discomfort appeared from the usage of the VR as well as the desktop display.

The data were analyzed using Statistical Package for Social Science Software (SPSS) (version 12 for Windows, SPSS, Inc., Chicago, IL, USA). The normality of the data was analyzed using Shapiro-Wilk normality test. The data of TMH, NIKBUT and SSQ were normally distributed, paired sample t-test was used in order to compare the parameters that were measured before and after using devices respectively. There was also comparison between the VR and laptop after 30 minutes of usage by using paired sample t-test.

\section{RESULTS}

\section{Comparing means of Tear Stability parameters (NIKBUT and TMH) before and after the usage of VR and Laptop}

The significance levels and the difference pre- and post-usage of VR and Laptop on tear stability.

Paired t-test analysis showed that all the p-values for both NIKBUT as well as TMH on both eyes were $>0.05$, indicated that there was no significant difference in the participants' tear stability before and after the usage of VR and Laptop.

As for the mean difference of NIKBUT, VR showed reduction in the range of $-1.16 \mathrm{~s}$ to $-2.03 \mathrm{~s}$, while contrary for the laptop, it showed increment of tear break up time from $0.2 \mathrm{~s}$ to $0.16 \mathrm{~s}$. However, the reduction of VR's NIKBUT was not statistically significant as the $\mathrm{p}$-values were $>0.05$. Similarly, for the mean difference of TMH, both different types of visual experience showed decrement in the range of 0.01 to 0.03 , but the changes did not give any significant difference to the tear film stability. Although all $\mathrm{p}$-values from all parameters were not statically significant, comparing between VR and laptop, the data for both NIKBUT and TMH showed more promising result for VR as it was near significant level. Table 1 shows the summary of significance levels and the difference of pre- and post-usage from this study.
When comparing the results of tear stability between the VR and laptop, there was also no significant changes as the p-value showed higher than the significant level for both eyes $(p>0.05)$, means that there is no clinically meaningful data. All the VR data showed higher mean value compared with laptop, except for the NIKBUT on the right eye and TMH on the left eye. Table 2 shows the summary of the significant level when comparing the changes of both devices on both eyes.

\section{Comparing the discomfort (SSQ-Total) after the usage of VR and Laptop}

For SSQ measurement, it was quantified based on [14] guidelines. The comparison of ocular discomfort was made between both visual experience of VR and laptop. The total score for SSQ or SSQ-Total was calculated to determine overall discomfort of the VR usage.

The significance levels and the difference pre- and post-usage of VR and laptop on the ocular discomfort

Paired t-test analysis showed that all the p-values for SSQ-Total on both devices, before and after, were more than 0.05 , indicated that there was no significant difference in the discomfort of the eyes after the usage of VR and laptop, as shown in Table 3. The mean values of SSQ-Total were higher after the use of both devices, however, the result was insignificant, VR showed higher mean value than the laptop [VR-Post:

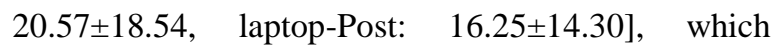
indicated that the VR give more discomfort compared with the laptop.

\section{DISCUSSION}

Effect of 3D image on the tear film stability and ocular discomfort

In this study, the data of 2D visual experience from the laptop and 3D visual experience from the virtual reality headset were compared before and after the use of both devices, in order to evaluate the effect of VR on the tear stability as well as quantify its comfort after 30 minutes. 
Mohd Zulfaezal Che Azemin etl. al./ Journal of Engineering and Science Research, 4(1) 2020, Pages: $40-46$

Table 1 Paired t-test comparison of NIKBUT and TMH before and after 30 minutes of watching movie using different visual image.

\begin{tabular}{ccccccccc}
\hline $\begin{array}{c}\text { Visual } \\
\text { Experienc } \\
\mathbf{e}\end{array}$ & $\begin{array}{c}\text { Measured } \\
\text { eye }\end{array}$ & $\begin{array}{c}\text { Time of } \\
\text { Measurem } \\
\text { ent }\end{array}$ & $\begin{array}{c}\text { NIKBUT } \\
\text { Mean } \pm \text { SD } \\
\text { (seconds) }\end{array}$ & t-value & p-value & $\begin{array}{c}\text { TMH } \\
\text { Mean } \pm \text { SD } \\
(\mathbf{m m})\end{array}$ & t-value & p-value \\
\hline \multirow{2}{*}{ VR } & Right & Pre & $9.99 \pm 5.48$ & 1.08 & 0.29 & $0.32 \pm 0.07$ & -1.99 & 0.06 \\
& & Post & $8.83 \pm 5.21$ & & & $0.29 \pm 0.09$ & & \\
& Left & Pre & $11.65 \pm 7.25$ & 1.15 & 0.14 & $0.30 \pm 0.08$ & -1.55 & 0.06 \\
& & Post & $9.62 \pm 5.00$ & & & $0.28 \pm 0.05$ & & \\
\hline \multirow{2}{*}{ Laptop } & Right & Pre & $8.94 \pm 4.99$ & -1.17 & 0.87 & $0.31 \pm 0.08$ & -1.93 & 0.13 \\
& & Post & $9.14 \pm 6.47$ & & & $0.29 \pm 0.06$ & & \\
& Left & Pre & $9.62 \pm 5.00$ & -0.15 & 0.88 & $0.30 \pm 0.09$ & -0.83 & 0.41 \\
\hline
\end{tabular}

Table 2 Paired t-test comparison of post VR and laptop after 30 minutes of watching movie for TMH and NIKBUT.

\begin{tabular}{cccccccc}
\hline $\begin{array}{c}\text { Measured } \\
\text { eye }\end{array}$ & $\begin{array}{c}\text { Types of } \\
\text { devices } \\
\text { used }\end{array}$ & $\begin{array}{c}\text { NIKBUT } \\
\text { Mean } \pm \text { SD } \\
\text { (seconds) }\end{array}$ & t-value & p-value & $\begin{array}{c}\text { TMH } \\
\text { Mean } \pm \text { SD } \\
(\mathbf{m m})\end{array}$ & t-value & p-value \\
\hline Right & VR & $8.83 \pm 5.21$ & -1.07 & 0.29 & $0.29 \pm 0.09$ & 0.432 & 0.67 \\
& Laptop & $9.14 \pm 6.47$ & & & $0.29 \pm 0.06$ & & \\
Left & VR & $11.65 \pm 7.25$ & 1.06 & 1.41 & $0.28 \pm 0.05$ & -0.731 & 0.47 \\
& Laptop & $9.78 \pm 5.23$ & & & $0.29 \pm 0.06$ & & \\
\hline
\end{tabular}

Table 3 Comparison of SSQ-Total before and after 30 minutes of watching movie using different visual image.

\begin{tabular}{ccccc}
\hline $\begin{array}{c}\text { Types of } \\
\text { devices }\end{array}$ & $\begin{array}{c}\text { Time of } \\
\text { Measurement }\end{array}$ & $\begin{array}{c}\text { SSQ-Total } \\
\text { Mean } \pm \text { SD }\end{array}$ & t-value & p-value \\
\hline VR & Pre & $17.41 \pm 16.85$ & -1.64 & 0.11 \\
& Post & $20.57 \pm 18.54$ & & \\
Laptop & Pre & $11.80 \pm 11.83$ & -2.35 & 0.25 \\
& Post & $16.25 \pm 14.30$ & & 0.16 \\
Post & VR & $20.57 \pm 18.54$ & 1.46 & \\
& Laptop & $16.25 \pm 14.30$ & & \\
& & &
\end{tabular}

The findings on tear stability did not show significant decrement of the NIKBUT after 30 minutes usage on both of devices, thus, suggesting no meaningful significant tear stability changes either on $3 \mathrm{D}$ or $2 \mathrm{D}$ visual experience. While, 2D visual experience showed an increment which was contrary from the expectation based on previous studies such as [15, 16, 17, 18]. These studies reported on the reduction of tear break up time using computer screen which is 2D image visualization. The slight reduction and increment of NIKBUT indicated that the tear stability was not affected even watching 3D with the HMD VR which is contradicts the general belief that using HMD will have side effect by reducing TBUT [11] or give tear film instability more than 2D image such as smartphone [4]. These findings also showed that extending the usage time did not affects the tear stability. Study by [19], did show that TBUT was not affected with the 10 minutes usage of computer screen, and when comparing with 30 minutes usage of both devices in this study, it seems that the TBUT was still not affected.

Meanwhile, the 30 minutes usage of the devices neither 2D nor 3D showed any significant effects to the TMH. All the post results of TMH were decreased, suggesting 
the decrement of the tear volume. This paper [20] reviewed that TMH is estimated to be a direct indicator for the tear volume test as it is agreed with the other diagnostic dry eye test such phenol red test, fluorescein tear break-up time and Schirmer's tests. The volume of the lower tear meniscus seemed to be increased by increment the amount of tear produced [21] and vice versa. Overall, in this study, the decrement of NIKBUT is proportional with the decrement of the $\mathrm{TMH}$, suggesting reduction of blinking rate. Theoretically, any activity that suppresses blinking such as in video display terminals (VDT) works, will interfere with the tear distribution [22]. The insufficient distribution of tears occurs with the decreased blinking, even when there is adequate tear production by lacrimal gland which may cause dry eye and discomfort [22]. As blinking rate reduced, the exposed ocular surface area is increased, which in the end causes the increment of tear evaporation [22] and affecting the tear stability which may give rise to symptoms of ocular discomfort. However in this study, the 3D and 2D image showed minimal effect on tear stability, but the presumed on decreasing tear stability effects of using a 3D display as compared with a 2D display [4] were not found and it seem that there was no different with the controlled devices.

In general, the discomfort score from the SSQ, did show higher total score on both post usage of devices, but there was not any meaningful significant effect to the subjective impression of ocular discomfort for both devices. This finding was opposite to the previous studies by [4, 5], but similarly, it was found in [11], suggesting 3D image did not give any discomfort effect to the user even with 30 minutes usage of VR continuously. The symptoms might be too small to be noticed by the some of the users depends on their sensitivity. SSQ indicators also was complicated to detect the symptoms due to rapid deterioration of symptoms [4]. The symptoms might be resolved rapidly after the use of the VR which in the end ignored by the users to be noted.

\section{Recommendations and suggestions for future research}

As not much study has been done on relating the after effect of VR with the eye performance, the future research is suggested to include the study of dry eye syndrome on the VR specifically for the dry eye patients, instead of finding tear change only. Besides, the battery of tests should be performed not only NIKBUT and TMH but with the inclusion of other tests such as fluorescein TBUT, NIBUT, Schirmer's test in order to improve the precision of data related with the ocular tears. The multiple measurements should also be taken for each parameter to reduce the errors in future study.

In addition, to improve the data collection in future research, it is suggested to have only one optometrist to do the assessment for the tear stability especially on TMH measurement because it is very subjective which is determined manually based on the image taken using the Oculus K5M. The wearing time of VR usage in future study also should be increased to more than 30 minutes, because it is claimed that the majority of the VR consumer use the gadget longer than that, thus the symptoms of discomfort might appear if the user experiencing the 3D image more than 30 minutes [23].

\section{CONCLUSION}

Results of this study showed that the VR headset did not give any short-term effects in term of tear stability and ocular discomfort after 30 minutes. Instead, neither VR nor laptop showed any effect on the eyes of wearer even when we quantified the symptoms using SSQ systematically. Previous studies did hypothesize that the VR might give dryness to the eye based on the 2D findings as it can alter the tear stability and give discomfort to the wearer. However, it seems that, the findings on this study is opposite as expected, thus positively convincing the society on its safety even when it is used for a half an hour. We also attempt to show to the consumers how tear stability and ocular discomfort caused by HMD is comparable to conventional 2D digital screen standards. In general, our results suggest that VR HMD wear may not provide a dryness-inducing stimulus despite the close viewing distances and long wearing hour involved. Currently, HMD and VR are still in their development phase, and the research needs to be continued into monitoring the different types of the VR's symptoms based on different age, ethnic, visual games and variations in specification. 


\section{ACKNOWLEDGEMENT}

Special thanks and gratitude to the co-researchers Mohamad Syarifuddin Bin Mukhtar, Anas Bin Ibrahim and Hafizah Binti Zaini, which helped during the data collection in this study. The author would also like to express thanks to all participants involved in this study. Their contributions are sincerely appreciated and gratefully acknowledged. This work is supported by the International Islamic University Malaysia (IIUM), under the Research Initiative Grant (P-RIGS18-0350035).

\section{REFERENCES}

[1] Fuchs, H. and Bishop, G. 1992. Research Directions in Virtual Environments. NFS Invitational Workshop, Univ. North Carolina, Retrieved from: https://www.ijser.org/researchpaper/Brief-

Introduction-of-Virtual-Reality-its-Challenges.pdf.

[2] Turnbull, P.R.K. and Philips, J.R. 2017. Ocular Effects of Virtual Reality Headset Wear In Young Adults. Scientific Reports, DOI: 10.1038/s41598017-16320-6.

[3] Thompsom, S. 2019. VR Applications: 21 Industries already using Virtual Reality. Virtual Speech, Retrieved from: https://virtualspeech.com/blog/vrapplications.

[4] Han, J. 2017. Comparison of Visual Discomfort and Visual Fatigue between Head-Mounted Display and Smartphone. Human Vision and Electronic Imaging, DOI:10.2352/ISSN.2470-1173.2017.14.HVEI-146.

[5] Sharples, S., Cobb, S., Moody, A. and Wilson, J.R. 2008. Virtual reality induced symptoms and effects (VRISE): Comparison of head mounted display (HMD), desktop and projection display systems. $\begin{array}{llll}\text { Displays, } & 29, & 2, & 58-69 \text {, }\end{array}$ DOI:10.1016/j.displa.2007.09.005.

[6] Prabhasawat, P., Pinitpuwadol, W., Angsriprasert, D., Chonpimal, P. and Salman, M. 2018. Tear film change and ocular symptoms after reading printed book and electronic book: a crossover study. Japanese Journal of Ophthalmology, https://doi.org/10.1007/s10384-018-00648-1.

[7] Jaiswal, S., Asper, L., Long, J., Lee, A., Harrison, K. and Golebiowski, B. 2019. Ocular and visual discomfort associated with smartphones, tablets and computers: what we do and do not know. Clin Exp Optom., DOI:10.1111/cxo.12851.

[8] Murube, J., Murube, E. 2002. Near Vision Accommodation in Horizontality with Vdt: Why Low Blinking and Dry Eye? Advances in
Experimental Medicine and Biology, 506, Pt B, 1205-1211, DOI: 10.1007/978-1-4615-0717-8_69.

[9] Sheehy, J.E. and Wilkinson, M. 1989. Depth perception after prolonged usage of night vision goggles. Aviat Space Environ Med., 60, 573-579.

[10] Wan, J.P., Rushton, S., and Mon-Williams, M. 1994. Binocular vision in a virtual world: visual deficits following the wearing of a head-mounted display. Ophthalmic Physiol Opt., 13, 4, 387-391.

[11] Peli, E. 1997. The visual effects of head-mounted display (HMD) are not distinguishable from those of desk-top computer display. Vision Research, 38, 2053-2066, DOI: 10.1016/S0042-6989(97)00397-0.

[12] Koh, S, Ikeda, C, Fujimoto, H, Oie, Y, Soma, T, Maeda, N. and Nishida, K. 2016. Regional Differences in Tear Film Stability and Meibomian Glands in Patients With Aqueous-Deficient Dry Eye. Eye Contact Lens, 42, 4, 250-255, DOI: 10.1097/ICL.0000000000000191.

[13] Koh, S., Ikeda, C., Watanabe, S., Oie, Y., Soma, T, Watanabe, H., Maeda, N. and Kohji 2014. Effect of non- invasive tear stability assessment on tear meniscus height. Acta Opthalmologica, 93, 2, 135139, https://doi.org/10.1111/aos.12516.

[14] Kennedy, R.S., Lane, N.E., Berbaum, K.S. and Lilienthal, M.G. 1993. Simulator Sickness Questionnaire: An Enhanced Method for Quantifying Simulator Sickness. The International Journal of Aviation Psychology, 3, 3, 203-220, DOI:10.1207/s15327108ijap0303_3.

[15] Cardona, G., García, C., Serés, C., Vilaseca, M. and Gispets, J. 2010. Blink Rate, Blink Amplitude, and Tear Film Integrity during Dynamic Visual Display Terminal Tasks. Current Eye Research, 36, 3, 190197, DOI: $10.3109 / 02713683.2010 .544442$.

[16] Hirota, M., Uozato, H., Kawamorita, T., Shibata, Y. and Shinya, Y. 2013. Effect of Incomplete Blinking on Tear Film Stability. Optometry and Vision Science, $\quad 90, \quad 7, \quad 650-657, \quad$ DOI: 10.1097/OPX.0b013e31829962ec.

[17] Wu, H., Wang, Y., Dong, M. and Yang, F. 2014. Meibomian gland dysfunction determines the severity of the dry eye conditions in visual display terminal workers. ARVO journals, DOI: 10.1371/journal.pone.0105575.

[18] Yazici, A., Sari, E.S., Sahin, G. and Kilic, A. 2015. Change In Tear Film Characteristics In Visual Display Terminal Users. European Journal of Ophthalmology, $\quad 25, \quad 85-89, \quad$ DOI: 10.5301/ejo.5000525.

[19] Patel, S., Henderson, R., Bradley, L., Galloway, B. and Hunter, L. 1991. Effect of VDU on Blinking rate and tear stability. Optom. Vis. Sci., 68, 11, 888-892, DOI: 1040-5488/91/6811-0888\$03.00/0.

[20] Minestone, C.J., Bruce, A.S. and Golding, T.R. 1996. Tear meniscus measurement in the diagnosis 
of dry eye. Current Eye Research, 15, 6, 653-661, DOI:10.3109/02713689609008906.

[21] Scherz, W., Doane, M.G. and Dohlman, C.H. 1974.Tear Volume in Normal Eyes and Keratoconjunctivitis sicca. Klin. Ophthalmol., 192, 141-150, https://doi.org/10.1007/BF00410700.

[22] Tsubota, K. 1998. Tear Dynamics and Dry Eye. Progress in Retinal and Eye Research, 17, 4, 565596, DOI: 10.1016/S1350-9462(98)00004-4.

[23] n. a. (2019). Virtual reality and augmented reality (VR and AR) devices average session time in the United States as of 2018 in minutes. Statista, Retrieved from: https://www.statista.com/statistics/831819/usvirtual-augmented-reality-device-average-sessiontime. 